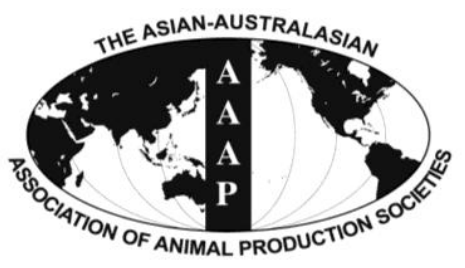

Asian Australas. J. Anim. Sci.

Vol. 26, No. 5 : 609-624 May 2013

http://dx.doi.org/10.5713/ajas.2012.12398

www.ajas.info

pISSN 1011-2367 elSSN 1976-5517

\title{
Microarray Analysis of Genes Involved with Shell Strength in Layer Shell Gland at the Early Stage of Active Calcification
}

\author{
Zhangguo Liu ${ }^{1,2, a}$, Qi Zheng ${ }^{3, a}$, Xueyu Zhang ${ }^{4}$ and Lizhi Lu ${ }^{5, *}$ \\ ${ }^{1}$ The Nurturing Station for the State Key Laboratory of Subtropical Silviculture, \\ Zhejiang A \& F University, Lin'an, Zhejiang, 311300, China
}

\begin{abstract}
The objective of this study was to get a comprehensive understanding of how genes in chicken shell gland modulate eggshell strength at the early stage of active calcification. Four 32-week old of purebred Xianju hens with consistent high or low shell breakage strength were grouped into two pairs. Using Affymetrix Chicken Array, a whole-transcriptome analysis was performed on hen's shell gland at $9 \mathrm{~h}$ post oviposition. Gene ontology enrichment analysis for differentially expressed (DE) transcripts was performed using the web-based GOEAST, and the validation of DE-transcripts was tested by qRT-PCR. 1,195 DE-transcripts, corresponding to 941 unique genes were identified in hens with strong eggshell compared to weak shell hens. According to gene ontology annotations, there are 77 DE-transcripts encoding ion transporters and secreted extracellular matrix proteins, and at least 26 DE-transcripts related to carbohydrate metabolism or post-translation glycosylation modification; furthermore, there are 88 signaling DE-transcripts. GO term enrichment analysis suggests that some DE-transcripts mediate reproductive hormones or neurotransmitters to affect eggshell quality through a complex suite of biophysical processes. These results reveal some candidate genes involved with eggshell strength at the early stage of active calcification which may facilitate our understanding of regulating mechanisms of eggshell quality. (Key Words: Chicken, Microarray, Differentially Expressed Genes, Eggshell Strength)
\end{abstract}

\section{INTRODUCTION}

The chicken eggshell is a porous bioceramic container which protects the egg against physical damage and microbial contamination. Avian eggshell consists of the innermost bilayered membranes, a calcified layer composed of a mamillary and pallisade layer, and the outermost cuticle. The calcified layer consists of both inorganic minerals and extracellular matrix. It is well known that the shell mineral amount (thickness or density) is the main

\footnotetext{
* Corresponding Author: Lizhi Lu. Tel: +86-571-86406682, Fax: +86-571-86406682, E-mail: Lulizhibox@163.com

${ }^{2}$ College of Animal Science, Zhejiang A. \& F. University, Lin'an, Zhejiang, 311300, China.

${ }^{3}$ Department of Biology, 104 Lynch Laboratory, University of Pennsylvania, Philadelphia, PA 19104, USA.

${ }^{4}$ Institute of Poultry Science, Chinese Academy of Agricultural Science, Yangzhou, Jiangsu, 225003, China.

${ }^{5}$ Institute of Animal Husbandry and Veterinary Science, Zhejiang Provincial Academy of Agricultural Science, Hangzhou, Zhejiang, 310021, China.

a These authors contributed equally to this work.

Submitted Jul. 18, 2012; Accepted Sept. 15, 2012; Revised Oct. 8, 2012
}

factor contributing to the mechanical properties of the eggshell (Ahmed et al., 2005). However, the organic matrix, although its content in the calcified layer is only 2 to $3.5 \%$, is of great importance to the deposition of bicarbonate and calcium ions, and to eggshell strength by controlling calcite crystal nucleation, growth, size and orientation (Greenfield et al., 1984).

The organic matrix in the calcified layer is comprised of a complex suite of components. In the acid soluble part of chicken eggshell matrix, 520 proteins have been identified (Mann et al., 2006), including several abundant proteins such as ovalbumin (Hincke, 1995), ovotransferrin (Gautron et al., 2001b), lysozyme (Hincke et al., 2000), osteopontin (Pines et al., 1995), sialoprotein (Solomon, 1999), clusterin (Mann et al., 2003), ovocleidin-17 (Hincke et al., 1995), ovocleidin-23 (Mann, 1999), ovocleidin-116 (Carrino et al., 1997), ovocalyxin-32 (Gautron et al., 2001a) and ovocalyxin-36 (Gautron et al., 2007). Many of the above components have been reported to undergo various posttranslation modifications, which allow them to be effective chelators for interacting with the inorganic materials (Veis, 1989; Reyes-Grajeda et al., 2004; Mann et al., 2007), or to 
mediate protein-protein interactions to facilitate the assembly of the organic matrix (Lakshminarayanan et al., 2002; Ney et al., 2006).

It has been demonstrated that some genes in hen oviduct are associated with eggshell formation, whose expression is dependent on mechanical strain (Pines et al., 1995; Lavelin et al., 1998; Lavelin et al., 2002). It is proposed that some genes may function as crucial modulators for eggshell quality through regulating signal transduction, ion transportation, expression or modification of organic components, and many other processes. However, despite the importance of eggshell strength in the poultry industry, very few transcriptome-wide studies regarding this trait have been published to date (Yang et al., 2007; Dunn et al., 2009; Jonchère et al., 2010).

It is well documented that various parts of the avian eggshell are formed in specific regions of the oviduct as the egg passes through them. During the laying sequence, about $4 \mathrm{~h}$ after previous oviposition, the next egg arrives at and will take about $1 \mathrm{~h}$ to pass through the white isthmus, in which the bilayered shell membranes are built around the egg. Then the egg enters the initial part of the shell gland, the red isthmus (tubular shell gland), and stays there for about $5 \mathrm{~h}$ to form mammillary knobs (Reyes-Grajeda et al., 2004). Finally the egg reaches the uterus (the main part of shell gland) and stays for an additional $15 \mathrm{~h}$ to form the palisade layer (Creger et al., 1976). It is known that the mamillary layer is the base of calcite crystal nucleation and crystal growth, and the palisade layer is the main part of the calcified shell, both of which affect global eggshell quality (Reyes-Grajeda et al., 2004; Jonchère et al., 2010).

In this study, we focused on the shell gland (uterus tissue near the red isthmus) at about $9 \mathrm{~h}$ post oviposition (corresponding to the early stage of active calcification, or to the transition stage from mammillary knob formation to construction of the palisade layer), and identified differentially expressed genes (DE-genes) in the layers with high shell strength compared to those with weak eggshell. Our results provide insight into the candidate genes involved in the mamillary layer formation and calcification that is crucial to the mechanical properties of avian eggshells.

\section{MATERIALS AND METHODS}

\section{Animal treatments}

Ninety purebred Xianju hens (a widely-bred Chinese indigenous chicken breed) of 28 weeks old were individually housed in laying cages. Birds were maintained under a cycle of $16 \mathrm{~h}$ light and $8 \mathrm{~h}$ dark. All birds were fed ad libitum with water and a mash layer diet (165 g protein, $35 \mathrm{~g} \mathrm{Ca}, 11.29 \mathrm{MJ} \mathrm{ME} / \mathrm{kg}$, as recommended by NRC of China, 2004).
After $10 \mathrm{~d}$ of adaptation for hens, the oviposition time of each egg was initiated to be observed and recorded, then egg weight and shape index (length/width) were measured immediately. Following strength testing, the egg content was discarded and the shell was washed, dried at room temperature and weighed. Shell thickness without membranes was measured with a digital micrometer. Shell index $\left(\mathrm{g} / 100 \mathrm{~cm}^{2}\right)$ (Sauveur, 1988) was calculated as $\mathrm{I}=$ $(\mathrm{C} / \mathrm{S}) \times 100$, in which $\mathrm{C}$ is the weight of shell with membranes, $\mathrm{S}$ is the shell surface $\left(\mathrm{cm}^{2}\right)$ with $\mathrm{S}=4.68 \times \mathrm{P}^{2 / 3}$ where $\mathrm{P}=$ egg weight $(\mathrm{g})$. All above measurements were consecutively carried out daily for $16 \mathrm{~d}$.

Finally, 2 groups of 2 hens with consistent high or low shell breakage strength were found. The differences between the eggshell properties of the selected 4 hens were analyzed by One-way ANOVA variance analysis in SPSS statistic software.

The four hens of interest were humanely sacrificed about $9 \mathrm{~h}$ after the previous oviposition. It is of note that all of the sacrificed hens had eggs in their uteruses (Figure 1A). The fat was removed from the uterus tissues near red isthmus and the tissues were then frozen in liquid nitrogen immediately and stored at $-80^{\circ} \mathrm{C}$. The animal treatments were approved by the Commission for Animal Welfare of Zhejiang A\&F University.

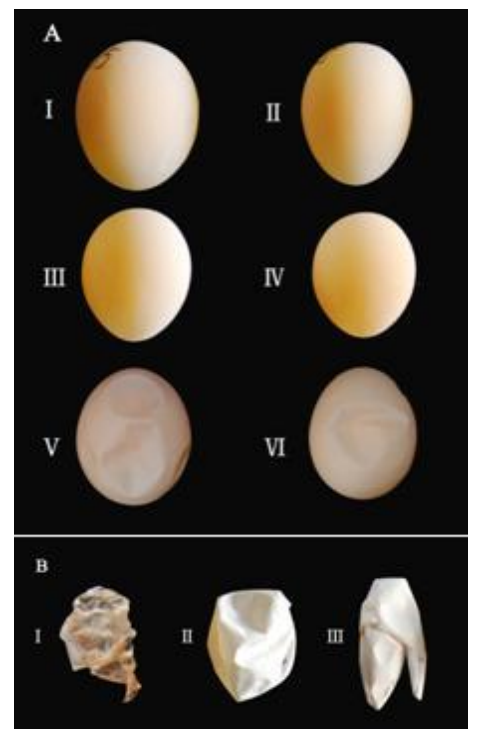

Figure 1. Eggs and forming eggshells obtained from hen shell glands at the moment of tissue sampling. Figure 1A: Eggs obtained from the shell glands of hens when sampling uterus tissues. A-I is the egg from \#35 hen, A-II from \#19 hen, A-III from $\# 80$ hen, A-IV from \#40 hen; these hens were all slaughtered at about $9 \mathrm{~h}$ after previous oviposition (P.O.). While A-V and A-VI eggs are from another 2 hens culled at 11.5 to $12 \mathrm{~h}$ after P.O., respectively. Figure 1B: The forming "shell" sampled at different stage of eggshell formation. B-I is the "shell" from the hen slaughtered at $4 \mathrm{~h}$ after P.O.; B-II is the "shell" of above egg from \#40 hen. B-III is the "shell" sampled at $12 \mathrm{~h}$ post oviposition. 


\section{Measurement of eggshell strength}

After egg weight and shape index measurements, the uncracked fresh eggs were individually placed lengthways with its blunt end upward in the FHK testing machine (Fujihara Co., Tokyo, Japan), and the vertical pressure was increasingly loaded upon the eggshell until the eggshell cracked and the eggshell strength was recorded as the maximum load (kgf).

\section{RNA preparation}

About $500 \mathrm{mg}$ of the tissue of the uterus near red isthmus, including the mucosa, muscularis and outer serosa was powdered under liquid nitrogen. The total RNA was extracted using the RNAiso Plus Mini Kit (TaKaRa, Dalian, P.R. China) according to the manufacturer's instructions. RNA concentration and purity were measured by a NanoDrop spectrophotometer (NanoDrop ${ }^{\circledR}$ ND-1000, NanoDrop Technologies, DE).

\section{Microarray hybridization and image acquisition}

Microarray analysis was performed by the Bioassay Laboratory of CapitalBio Corporation (CapitalBio Co., Beijing, China). Briefly, the RNA integrity was firstly assessed using a Bioanalyzer (Agilent Technologies, Cheshire, UK), then $2 \mu \mathrm{g}$ of total RNA was used for reverse transcription and biotin-labeled cRNA synthesis according to the manufactures' instructions, and finally subjected to microarray hybridization. The Affymetrix GeneChip ${ }^{\circledR}$ Chicken Genome Array (Affymetrix, Santa Clara, CA, USA) was used in this study, which contains 38,535 probesets corresponding to $>28,000$ chicken genes. Following $16 \mathrm{~h}$ of hybridization, the arrays were immediately washed, stained and scanned using Affymetrix ${ }^{\circledR}$ GeneChip ${ }^{\circledR}$ scanner 3000 (Affymetrix, Santa Clara, CA, USA), and the image files were processed into raw CEL intensity files using GeneChip Operating Software (GCOS version 1.2).

\section{Pre-processing and normalization of microarray data}

The raw intensity files generated by GCOS were imported and processed by $\mathrm{R}$ with Bioconductor packages. The total RNA quality was firstly verified statistically again by plotting the 5'-3' hybridization signal trends across all target transcripts. Then the microarray intensity was processed into transcript expression by the Affymetrix MAS5.0 method implemented in the $\mathrm{R}$ package, a procedure including background normalization, $\mathrm{PM} / \mathrm{MM}$ probe correction, expression summarization and constant normalization on probeset level.

\section{Identification of DE-transcripts}

To identify DE-transcripts, the 4 array samples were first grouped into two pairs of high vs. low eggshell strength according to eggshell property differences of the hens (see results). According to Cheuk and Cheng (2011), Affymetrix platform is relatively precise and sensitive in detecting signals, the DE-transcripts were identified as those with fold-change $>=2$ in either of the two pairs of comparison and a statistical significant difference between high strength and low strength samples $(\mathrm{p}<0.05$, Welch $\mathrm{t}-$ test). It is of note that the log-odds values (Lods) of expression fold-change were used in the analysis; therefore, the DE-transcripts always have an absolute Lods value no less than $1(|\operatorname{Lods}| \geq 1)$.

\section{Gene ontology enrichment analysis}

Gene ontology enrichment analysis for DE-transcripts was performed using the web-based GOEAST (Zheng and Wang, 2008) Affymetrix analysis tool, with FDR cut-off of 0.05 using Yekutieli's FDR adjustment method.

\section{Validation of differential expression by qRT-PCR experiments}

Twenty-one DE-transcripts, with a fold-change ranging from low to high, were selected for further validation with qRT-PCR experiments; and all two groups of microarray samples were tested. Glyceraldehyde 3-phosphate dehydrogenase $(G A P D H)$ was used as the internal reference in all the PCR experiments. The primer sequences for qRTPCR experiments can be found in Table 1.

To begin, total RNA was individually reverse transcribed with the $\mathrm{SYBR}^{\circledR}$ PrimeScript $^{\mathrm{TM}}$ RT-PCR kit II (TaKaRa, Dalian, China) according to the manufacturer's instructions. Then above RT-PCR kit was further used for fluorescence detection on an ABI Prism 7500 Sequence Detection System (Applied Biosystems, USA). All samples were analyzed in triplicates.

Dissociation curve analysis was conducted to ensure that a single PCR product with appropriate size was amplified in each reaction. On the other hand, the examination of PCR efficiency was performed based on LinRegPCR program (12.X) (Ramakers et al., 2003; Ruijter et al., 2009) to ensure internal and target transcript primers were amplified with similar efficiency.

The differential expression levels ( $\log 2$ units) were calculated using the equation $\log 2$ units (high versus low) $=$ $-\Delta \Delta \mathrm{Ct}$, where $\Delta \Delta \mathrm{Ct}=\left(\mathrm{Ct}^{\mathrm{th}}-\mathrm{Ct}^{\mathrm{ih}}\right)-\left(\mathrm{Ct}^{\mathrm{tl}}-\mathrm{Ct}^{\mathrm{il}}\right) . \mathrm{Ct}$ is the threshold cycle number when the amount of amplified product reaches a stable threshold. $\mathrm{Ct}^{\text {th }}$ and $\mathrm{Ct}^{\text {ih }}$ represented the $\mathrm{Ct}$ of target transcript and internal reference transcript of "high eggshell strength sample", respectively. Correspondingly, $\mathrm{Ct}^{\mathrm{tl}}$ and $\mathrm{Ct}^{\mathrm{il}}$ represented the $\mathrm{Ct}$ of target transcript and inner-reference transcript of "low eggshell strength sample", respectively. 
Table 1. Descriptions of specific primers used for real-time RT-PCR

\begin{tabular}{|c|c|c|c|c|}
\hline Gene symbol & Accession no. & Forward primer (5'-3') & Reverse primer (5'-3') & Amplicon (bp) \\
\hline$\overline{A C Y P 2}$ & XM_419292 & CGGCTCGCTCAAGTCGGTGG & GGCCCTGAACTTGGCCCGTC & 152 \\
\hline$A M D H D 1$ & XM_416158 & GCACTGGGAAGTGCGTATTGCCA & TCTTCCGTGGCCTTCCTGGTGT & 175 \\
\hline ATP6VIA & NM_204974 & TGCAACATGGCAGGTGCTGCT & TGCCAGGCCCCAGTTCCACT & 187 \\
\hline$C A 5 B$ & XM_414195 & CAGCTTGGCCACCTGCACTCC & ACACGTCGCTGGGTCGTAGCT & 175 \\
\hline CHST3 & NM_205121 & TGATGGCCACCACACGCACC & CTGCAGCACGTCGCGGTACA & 170 \\
\hline COL12A1 & NM_205021 & AGGCGAGTCTTCCCCGACGG & GCGCTGTCCTCATGTCTGCCC & 171 \\
\hline$C R A B P 1$ & NM_001030539 & CGCCCCGCCATGCCTAACTT & AACTGGTCCCCGTCCTGGCG & 161 \\
\hline$C R Y B B 1$ & NM_204180 & ACCTGGCGGACTGCGGGTT & CGGTAGCTGCTGGACCAGGTG & 151 \\
\hline ЕХОССВ & XM_420892 & AACCCCACCACAGCCCTCGT & TGGCTGTTGATGAGGCCGCG & 149 \\
\hline$F G B$ & XM_420369.2 & GCTGCTCCTGCTGCTCCTGC & GTGCCACGGGCCTGAGTGTG & 155 \\
\hline GAS2L3 & XM_416172 & GGAGTAGTGCTGGCAGTCCTGC & CCTGGGCCGTGTCTGGGAGT & 193 \\
\hline GIT2 & NM_204206 & TCGCTTGCCATGCCGTGAGG & GCAACGTGGAGCGGGGTGTT & 168 \\
\hline MAN1A2 & XM_416490 & ACGTGGACACCAGCAAGGGGG & TCCTTTGCCTCTTCCAGGGCCTTT & 148 \\
\hline NDST4 & XM_420638 & CGAGCAGCTTCCCTCATCCCCAA & TGCCCAGGGGCTTGACGTAA & 156 \\
\hline$N P Y$ & NM_204587 & GAGGACGCTCCCGCAGAGGA & TCGAAGGGTCTTCAAACCGGGA & 175 \\
\hline OC416916 & XM_415207 & TGGAGGTGGAGCACAAACATCTGC & CCACCGAGCACACAGCCAGAAA & 200 \\
\hline$P L C X D 1$ & NM_001128637 & CCTGGCCTGCAGGAATTTTGATGG & AGCCACGCTGCCACATGGTC & 137 \\
\hline RCJMB04_34k20 & NM_001031112 & GGACAGGCGGGCGAGAGAGT & TGGTGGTAACACGCACGCTGA & 126 \\
\hline$S L C 8 A 1$ & XM_415002 & CGTGTTTGTGGCACTGGGGACA & ATGGCCGCGATGGACCAAGC & 159 \\
\hline$T B X A S 1$ & XM_416334 & TGTGTGGTGCTGGGACAGCGT & ATACAGCCACGGGGTCCTGCT & 188 \\
\hline WDR72 & XM_425069 & GGCTGTTATCAGGGGGCCAGGA & GCACACGCAGCACACTACGC & 161 \\
\hline$G A P D H$ & NM_204305 & GGGCTGCTAAGGCTGTGGGG & TCAGGGGCCCATCAGCAGCA & 177 \\
\hline
\end{tabular}

\section{RESULTS}

\section{Eggshell quality of hens under study}

Among 90 tested hens, only 6 of them laid eggs at a similar laying rate with consistent high eggshell strength (defined as $\geq 4.5 \mathrm{kgf}$ ) or low strength (defined as $\leq 3.5 \mathrm{kgf}$ ). Two of these 6 hens were sacrificed at about 11.5 to $12 \mathrm{~h}$ post oviposition, but the eggshells collected from the shell glands showed more calcification extent than expected (Figure 1A and 1B). To focus on the initial stage of active calcification, we decided to use the uterus tissues near red isthmus from the remaining 4 hens, namely \#19, \#35, \#40 and \#80, at about $9 \mathrm{hr}$ post oviposition. The eggs and eggshells harvested from these 4 hens at the moment of tissue sampling are shown in Figure 1A and Figure 1B. The eggshell breaking strength is also shown in Table 2 for these 4 hens, with consistent high (\#19 and \#40) or low (\#35 and \#80) eggshell quality.

To eggs from \#19 and \#35 hens, the differences of both shell strength and shell weight were very significant $(\mathrm{p}<0.01$, t-test), but there was no significant difference ( $p>0.05)$ for other eggshell quality metrics, such as shell thickness or shell index (Table 2). On the other hand, the differences of all of above eggshell metrics between eggs from $\# 40$ and \#80 hens were very significant $(p<0.01)$ (Table 2). To get rigorous microarray data, therefore, we grouped the \#19 with \#35 hens due to the similarity of some of eggshell mechanical properties of the paired individuals; while \#40 and \#80 hens were also grouped as another pair.

\section{Differentially expressed transcripts}

The expression level of all probesets in 4 array samples were analyzed, and 1,195 DE-transcripts between uterus samples with high shell strength and low shell strength were identified. These DE-transcripts correspond to 941 unique genes. Among them, 407 genes were up-regulated in high strength samples comparing to low strength samples, and the other 534 genes were down-regulated. The expression

Table 2. Parameters related to eggshell quality of hens in this study

\begin{tabular}{lcccccc}
\hline Hen & shell strength (kgf) & shell thickness $(\mathrm{mm})$ & shell index $\left(\mathrm{g} / 100 \mathrm{~cm}^{2}\right)$ & shell weight $(\mathrm{g})$ & egg weight $(\mathrm{g})$ & shape index \\
\hline$\# 40$ & $5.17 \pm 0.40^{\mathrm{A}}$ & $0.367 \pm 0.016^{\mathrm{A}}$ & $8.07 \pm 0.28^{\mathrm{A}}$ & $4.297 \pm 0.144^{\mathrm{a}}$ & $38.46 \pm 1.52^{\mathrm{C}}$ & $1.274 \pm 0.024^{\mathrm{B}}$ \\
$\# 19$ & $4.75 \pm 0.21^{\mathrm{A}}$ & $0.328 \pm 0.012^{\mathrm{B}}$ & $7.18 \pm 0.25^{\mathrm{B}}$ & $3.968 \pm 0.195^{\mathrm{b}}$ & $40.72 \pm 1.26^{\mathrm{B}}$ & $1.322 \pm 0.030^{\mathrm{A}}$ \\
$\# 35$ & $2.99 \pm 0.71^{\mathrm{B}}$ & $0.324 \pm 0.023^{\mathrm{B}}$ & $6.97 \pm 0.68^{\mathrm{B}}$ & $4.363 \pm 0.426^{\mathrm{a}}$ & $48.60 \pm 1.38^{\mathrm{A}}$ & $1.278 \pm 0.032^{\mathrm{AB}}$ \\
$\# 80$ & $2.54 \pm 0.69^{\mathrm{B}}$ & $0.272 \pm 0.022^{\mathrm{C}}$ & $6.25 \pm 0.71^{\mathrm{C}}$ & $3.451 \pm 0.417^{\mathrm{C}}$ & $40.58 \pm 1.06^{\mathrm{B}}$ & $1.313 \pm 0.052^{\mathrm{AB}}$ \\
\hline
\end{tabular}

Values are from eggs laid by each hen of interest during the period of observation. Distinct capital letters in the same column indicate parameters between hens with a significant difference $(\mathrm{p}<0.01)$, and distinct small letters indicate the significant difference is at level $\mathrm{p}<0.05$. 


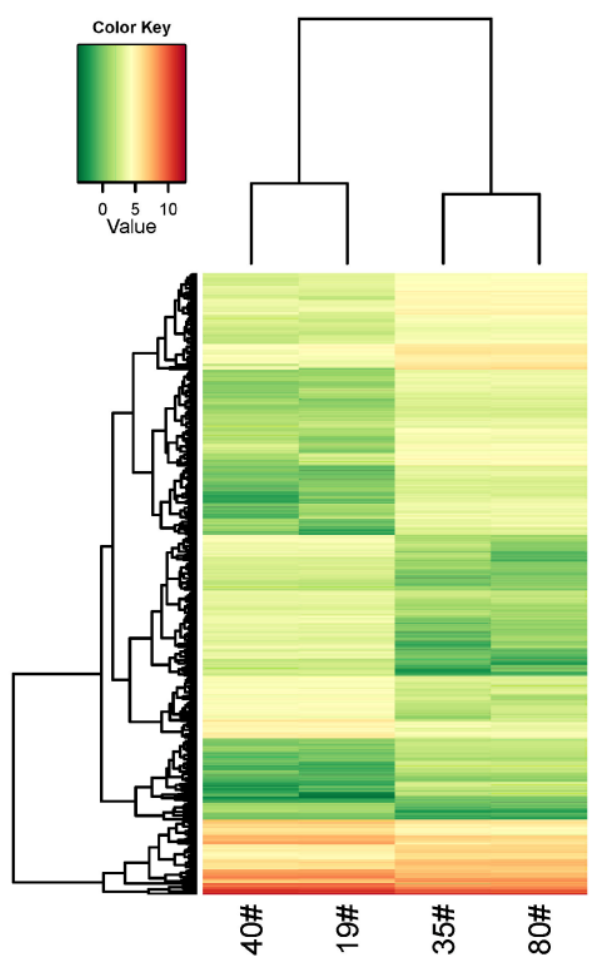

Figure 2. Heatmap and dendrogram of differentially expressed transcripts (DE-transcripts). Each cell represents the (normalized) gene expression value for given DE-transcript (row) in the specified sample (column). Cell colors indicate gene expression level: red: highly expressed; yellow: medium expression; green: lowly expressed. Row-side and column-side dendrogram represent the hierarchical clustering of DE-transcript expression for different transcripts or samples, respectively. Clustering is based on "complete-linkage" method using Euclidean-distance.

profile of all 1,195 DE-transcripts is shown in the heatmap in Figure 2. As shown in the heatmap, samples \#19 with \#40 and \#35 with \#80 were grouped as clusters among different samples, consistent with the similarity of their eggshell quality.
According to gene ontology annotations, the DEtranscripts are involved in a variety of biological processes. The most prominent DE-transcripts were found related to the following processes: signal transduction (88 DEtranscripts), ion transport and extracellular matrix organization (77 DE-transcripts), carbohydrate metabolism and protein modification (26 DE-transcripts) (Table 3).

Furthermore, avian calcified eggshell is a biomaterial composed of calcium salt and special ECM. The ECM is mainly comprised of collagens, glycoproteins and proteoglycans. Among the DE-transcripts, COL8A2, COL12A1, COL13A1, LOC424798, LAMA2, LAMA4, $L A M B 4$, and $L A M C 1$ may be related to extracellular matrix formation; while CHST3, GALNTL1, NDST4, LARGE, POFUT2, RCJMB04_28123, and MAN1A2 are all localized in the endoplasmic reticulum or Golgi apparatus, and likely mediate the processes of carbohydrate metabolism, or posttranslation glycosylation modification.

\section{Gene ontology (GO) term enrichment of DE-transcripts}

It is of note that although many DE-transcripts were found related to various biological processes according to their ontology annotations, they are not necessarily correlated to the eggshell quality, due to random noise or other non-specific confounding factors commonly existing in microarray or other high-throughput experiments. Therefore, using web-based GOEAST (Zheng and Wang, 2008) we further identified significantly enriched GO terms among all the DE-transcripts. According to biology processes or molecular functions, the enriched GO terms can be roughly classified into several groups (Tables 4 and 5).

A group of processes are involved in reproductive hormone regulation, which contain Somatotropin secreting cell differentiation (GO:0060126), adenohypophysis development (GO:0021984), and response to estradiol stimulus (GO:0032355) (Table 4).

Table 3. i) DE-transcripts related with signaling, ion transportation, extracellular matrix protein, and carbohydrate metabolism or posttranslation glycosylation modification

\begin{tabular}{lcccc}
\hline Gene symbol & Transcript ID & Log2 units (strong VS weak) & p-value & Category \\
\hline LOC771699 & XM_001234946 & 3.853 & 0.0004 & signaling \\
SH3PXD2A & XM_421741 & 3.410 & 0.0397 & signaling \\
LOC429955 & XM_427511 & 3.296 & 0.0034 & signaling \\
PDCL2 & XM_420702 & 3.100 & 0.0115 & signaling \\
LOC430487 & XM_428042 & 3.055 & 0.0074 & signaling \\
SEMA3G & XM_414289 & 2.933 & 0.0213 & signaling \\
RHOBTB2 & XM_001232709 & 2.905 & 0.0069 & signaling \\
RXFP1 & XM_420385 & 2.795 & 0.0205 & signaling \\
PIK3C2B & XM_417956 & 2.777 & 0.0194 & signaling \\
OR10A7 & XM_425093 & 2.768 & 0.0067 & signaling \\
NPY & NM_205473 & 2.401 & 0.0329 & signaling \\
PDE8B & XM_425218 & 2.401 & 0.0498 & signaling \\
GREM2 & XM_419552 & 2.284 & 0.0423 & signaling \\
\hline
\end{tabular}


Table 3. ii) DE-transcripts related with signaling, ion transportation, extracellular matrix protein, and carbohydrate metabolism or posttranslation glycosylation modification (Continued)

\begin{tabular}{|c|c|c|c|c|}
\hline Gene symbol & Transcript ID & Log2 units (strong VS weak) & p-value & Category \\
\hline$\overline{S P A G 9}$ & XM_420098 & 2.215 & 0.0423 & signaling \\
\hline LOC396365 & NM_205400 & 2.166 & 0.0009 & signaling \\
\hline$M A P K B P 1$ & XR_026772 & 2.157 & 0.0187 & signaling \\
\hline OXTR & NM_001031569 & 2.128 & 0.0134 & signaling \\
\hline STC2 & XM_414534 & 2.120 & 0.0135 & signaling \\
\hline$M P P 3$ & XM_418108 & 1.921 & 0.0491 & signaling \\
\hline GREM1 & NM_204978 & 1.903 & 0.0220 & signaling \\
\hline C20orf32 & XM_417499 & 1.889 & 0.0117 & signaling \\
\hline$C R A B P 1$ & NM_001030539 & 1.778 & 0.0115 & signaling \\
\hline Clorf107 & NM_001031051 & 1.770 & 0.0411 & signaling \\
\hline$R G S 9$ & XM_415685 & 1.657 & 0.0177 & signaling \\
\hline ARHGEF 12 & XM_417890 & 1.437 & 0.0014 & signaling \\
\hline LTBP3 & XM_426444 & 1.433 & 0.0409 & signaling \\
\hline$N G E F$ & NM_001010841 & 1.390 & 0.0307 & signaling \\
\hline PDE1A & XM_421969 & 1.363 & 0.0127 & signaling \\
\hline CRHBP & XM_424801 & 1.355 & 0.0159 & signaling \\
\hline SRGAPl & NM_001080101 & 1.335 & 0.0426 & signaling \\
\hline PDE9A & XM_416748 & 1.196 & 0.0475 & signaling \\
\hline NPFFR2 & NM_001034825 & 1.124 & 0.0283 & signaling \\
\hline WNT4 & NM_204783 & 1.114 & 0.0180 & signaling \\
\hline$F G D 4$ & XM_416365 & 1.099 & 0.0121 & signaling \\
\hline SOCS 2 & NM_204540 & 1.033 & 0.0377 & signaling \\
\hline TOB1 & NM_001001467 & 1.015 & 0.0347 & signaling \\
\hline$R N D 3$ & XM_422158 & 0.887 & 0.0094 & signaling \\
\hline ARHGAP28 & XM_419140 & -4.485 & 0.0231 & signaling \\
\hline WNT3 & NM_204675 & -3.902 & 0.0422 & signaling \\
\hline LOC428961 & NM_001142671 & -3.469 & 0.0058 & signaling \\
\hline$V A V 2$ & NM_204142 & -3.397 & 0.0232 & signaling \\
\hline$T B C 1 D 20$ & XM_001235014 & -3.154 & 0.0389 & signaling \\
\hline$H T T$ & XM_420822 & -3.086 & 0.0065 & signaling \\
\hline$P 2 R Y 2$ & XM_425667 & -3.079 & 0.0294 & signaling \\
\hline$G R A P 2$ & XM_001234081 & -2.967 & 0.0402 & signaling \\
\hline INPP4A & XM_416886 & -2.749 & 0.0130 & signaling \\
\hline ITSN1 & XM_416715 & -2.744 & 0.0139 & signaling \\
\hline$E D N R B 2$ & NM_204120 & -2.644 & 0.0317 & signaling \\
\hline$F G B$ & XM_420369 & -2.631 & 0.0194 & signaling \\
\hline$R X F P 3$ & XM_429217 & -2.616 & 0.0048 & signaling \\
\hline LOC420403 & XM_418509 & -2.531 & 0.0049 & signaling \\
\hline SSTR3 & NM_001024583 & -2.501 & 0.0203 & signaling \\
\hline GPR39 & NM_001080105 & -2.376 & 0.0087 & signaling \\
\hline GPR97 & XM_413998 & -2.356 & 0.0230 & signaling \\
\hline$F G F 14$ & NM_204777 & -2.322 & 0.0175 & signaling \\
\hline SFRP4 & XM_418831 & -2.204 & 0.0162 & signaling \\
\hline LOC430333 & XM_001235474 & -1.880 & 0.0153 & signaling \\
\hline GARNL1 & XM_421244 & -1.850 & 0.0477 & signaling \\
\hline RAPHI & XM_421961 & -1.842 & 0.0035 & signaling \\
\hline C14orf138 & XM_421460 & -1.756 & 0.0012 & signaling \\
\hline LOC421876 & XM_419893 & -1.750 & 0.0333 & signaling \\
\hline$N L E 1$ & XM_415857 & -1.735 & 0.0463 & signaling \\
\hline CHRM2 & NM_001030765 & -1.721 & 0.0310 & signaling \\
\hline
\end{tabular}


Table 3. iii) DE-transcripts related with signaling, ion transportation, extracellular matrix protein, and carbohydrate metabolism or posttranslation glycosylation modification (Continued)

\begin{tabular}{|c|c|c|c|c|}
\hline Gene symbol & Transcript ID & Log2 units (strong VS weak) & p-value & Category \\
\hline$\overline{L O C 768958}$ & XM_001232128 & -1.622 & 0.0278 & signaling \\
\hline RASLIOB & XM_001233673 & -1.546 & 0.0473 & signaling \\
\hline SIPA1L2 & XM_419564 & -1.545 & 0.0020 & signaling \\
\hline LOC769317 & XM_001231944 & -1.531 & 0.0287 & signaling \\
\hline$P L X D C 2$ & XM_418613 & -1.503 & 0.0155 & signaling \\
\hline SPOCK1 & XM_414622 & -1.491 & 0.0375 & signaling \\
\hline$C S F 2 R B$ & XM_001234608 & -1.468 & 0.0498 & signaling \\
\hline LOC429163 & XM_426718 & -1.434 & 0.0220 & signaling \\
\hline PLXNC1 & XM_416143 & -1.398 & 0.0103 & signaling \\
\hline RCJMB04_19g9 & XM_419989 & -1.339 & 0.0019 & signaling \\
\hline PLXNAI & XM_414370 & -1.305 & 0.0467 & signaling \\
\hline TSPAN5 & XM_420654 & -1.277 & 0.0142 & signaling \\
\hline LOC431251 & NM_001127171 & -1.226 & 0.0414 & signaling \\
\hline ANXA10 & XM_001233661 & -1.176 & 0.0441 & signaling \\
\hline RCJMB04_18c11 & NM_001012909 & -1.153 & 0.0436 & signaling \\
\hline SPRED2 & XM_419341 & -1.124 & 0.0146 & signaling \\
\hline$M O B K L 1 A$ & XM_420601 & -1.116 & 0.0026 & signaling \\
\hline$A L S 2 C L$ & XR_026875 & -1.038 & 0.0119 & signaling \\
\hline$M P P 1$ & NM_001007917 & -1.036 & 0.0424 & signaling \\
\hline$F G F 12$ & NM_204888 & -1.016 & 0.0006 & signaling \\
\hline GNA13 & XM_415686 & -1.007 & 0.0195 & signaling \\
\hline ARL10 & XM_414552 & -0.975 & 0.0492 & signaling \\
\hline$A D R A 2 B$ & XM_425203 & -0.885 & 0.0415 & signaling \\
\hline$C C K A R$ & NM_001081501 & -0.868 & 0.0352 & signaling \\
\hline RCJMB04_3n15 & NM_001030902 & -0.815 & 0.0373 & signaling \\
\hline$T B C 1 D 24$ & XM_001232296 & 4.629 & 0.0023 & IT \\
\hline SCN9A & XM_422021 & 3.745 & 0.0047 & IT \\
\hline KCNT2 & XM_426614 & 3.077 & 0.0015 & IT \\
\hline LOC395893 & & 3.030 & 0.0392 & IT \\
\hline NIPAL4 & XM_414566 & 2.796 & 0.0163 & IT \\
\hline ATP6VOA4 & NM_001080102 & 2.790 & 0.0397 & IT \\
\hline GRIN2B & XM_416204 & 2.781 & 0.0291 & IT \\
\hline KCNJ1 & XM_425795 & 2.363 & 0.0011 & IT \\
\hline POR & XM_415768 & 2.294 & 0.0047 & IT \\
\hline KCNK2 & XM_001234269 & 2.270 & 0.0447 & IT \\
\hline KIRREL3 & XR_026874 & 2.143 & 0.0193 & IT \\
\hline$G A B R G 2$ & NM_205345 & 1.985 & 0.0176 & IT \\
\hline SLC4A1 & NM_205522 & 1.794 & 0.0041 & IT \\
\hline NDUFA7 & XM_418185 & 0.995 & 0.0464 & IT \\
\hline JPH3 & XM_414192 & 0.925 & 0.0193 & IT \\
\hline CACNA2D1 & XM_001231265 & 0.852 & 0.0429 & IT \\
\hline EFCAB5 & XM_415833 & -3.593 & 0.0342 & IT \\
\hline SPATA22 & XM_001235167 & -3.590 & 0.0176 & IT \\
\hline RCJMB04_Ifl & NM_001031133 & -3.197 & 0.0328 & IT \\
\hline LOC428404 & XM_425965 & -3.177 & 0.0442 & IT \\
\hline ATP13A3 & XM_422709 & -3.059 & 0.0065 & IT \\
\hline$G A B R B 2$ & XM_001232377 & -2.963 & 0.0472 & IT \\
\hline SLC8A1 & NM_001079473 & -2.694 & 0.0342 & IT \\
\hline CACNA2D 3 & XM_414338 & -2.622 & 0.0100 & IT \\
\hline SERINC5 & XM 424762 & -2.615 & 0.0129 & IT \\
\hline
\end{tabular}


Table 3. iv) DE-transcripts related with signaling, ion transportation, extracellular matrix protein, and carbohydrate metabolism or posttranslation glycosylation modification (Continued)

\begin{tabular}{|c|c|c|c|c|}
\hline Gene symbol & Transcript ID & Log2 units (strong VS weak) & p-value & Category \\
\hline LOC421866 & XR_027148 & -2.613 & 0.0307 & IT \\
\hline LOC425295 & XM_423073 & -2.586 & 0.0212 & IT \\
\hline LOC772391 & XM_001235535 & -2.573 & 0.0163 & IT \\
\hline KCNK17 & XM_419477 & -2.506 & 0.0047 & IT \\
\hline KCTD16 & XM_425217 & -2.363 & 0.0076 & IT \\
\hline CNNM1 & XM_421703 & -2.309 & 0.0446 & IT \\
\hline KCNJ5 & XM_417864 & -2.138 & 0.0324 & IT \\
\hline RCJMB04_11e10 & NM_001030630 & -2.102 & 0.0226 & IT \\
\hline GRIN3A & XM_001232181 & -1.680 & 0.0355 & IT \\
\hline ATP6V1A & NM_204974 & -1.202 & 0.0065 & IT \\
\hline RCJMB04_16a12 & NM_001031305 & -1.030 & 0.0261 & IT \\
\hline CNGA3 & NM_205221 & -0.962 & 0.0479 & IT \\
\hline$P 2 R X 4$ & NM_204291 & -0.882 & 0.0252 & IT \\
\hline MEGF10 & XM_424719 & 3.910 & 0.0398 & EM \\
\hline FAT2 & XM_414584 & 3.892 & 0.0052 & EM \\
\hline$S D K 2$ & NM_204538 & 2.905 & 0.0097 & EM \\
\hline$N R X N 3$ & XM_421297 & 2.643 & 0.0139 & EM \\
\hline LAMA4 & XM_419780 & 2.569 & 0.0322 & EM \\
\hline NTNG1 & XM_001231446 & 2.004 & 0.0328 & EM \\
\hline CRTACI & NM_001080211 & 1.930 & 0.0132 & EM \\
\hline$L A M C 1$ & NM_204166 & 1.680 & 0.0257 & EM \\
\hline$L A M B 4$ & XM_001232877 & 1.642 & 0.0161 & EM \\
\hline PPFIA1 & XM_421074 & 1.583 & 0.0166 & EM \\
\hline CLDN20 & XM_001232002 & 1.438 & 0.0101 & EM \\
\hline $\mathrm{CDH} 9$ & XM_001231501 & 1.296 & 0.0311 & EM \\
\hline$C H A D$ & XM_416236 & 1.268 & 0.0240 & EM \\
\hline LOC396026 & NM_205128 & 1.239 & 0.0403 & EM \\
\hline PCDH21 & NM_001001759 & 1.174 & 0.0187 & EM \\
\hline$E P D R 1$ & XM_418830 & 1.158 & 0.0469 & EM \\
\hline CPNE8 & XM_001231388 & 1.121 & 0.0219 & EM \\
\hline COL12A1 & NM_205021 & 0.996 & 0.0003 & EM \\
\hline$P K P 2$ & XM_416362 & 0.983 & 0.0447 & EM \\
\hline$C D 72$ & NM_205052 & 0.855 & 0.0263 & EM \\
\hline NINJ2 & XM_416382 & -4.039 & 0.0409 & EM \\
\hline OTOF & XM_420015 & -3.980 & 0.0016 & EM \\
\hline COL13A1 & XM_001232218 & -3.260 & 0.0006 & EM \\
\hline SVEP1 & XM_424917 & -2.967 & 0.0250 & EM \\
\hline OTOP1 & XM_420790 & -2.830 & 0.0263 & EM \\
\hline$G P N M B$ & XM_425991 & -2.771 & 0.0327 & EM \\
\hline$P K P 1$ & XM_419240 & -2.349 & 0.0324 & EM \\
\hline$L A M A 2$ & XM_419746 & -2.336 & 0.0000 & EM \\
\hline COLSA2 & XM_425780 & -2.295 & 0.0170 & EM \\
\hline FNBP4 & XM_424260 & -2.279 & 0.0486 & EM \\
\hline EGFL6 & XM_416835 & -2.268 & 0.0392 & EM \\
\hline $\mathrm{CDH} 18$ & XM_426046 & -1.955 & 0.0090 & EM \\
\hline CLDN8 & XM_425544 & -1.598 & 0.0288 & EM \\
\hline RCJMB04_34k20 & NM_001031112 & -1.214 & 0.0085 & EM \\
\hline$S R P X$ & XM_416781 & -1.123 & 0.0244 & EM \\
\hline$D L G 1$ & XM_422701 & -1.084 & 0.0171 & EM \\
\hline FBLN1 & NM_204165 & -0.981 & 0.0206 & EM \\
\hline
\end{tabular}


Table 3. v) DE-transcripts related with signaling, ion transportation, extracellular matrix protein, and carbohydrate metabolism or posttranslation glycosylation modification (Continued)

\begin{tabular}{|c|c|c|c|c|}
\hline Gene symbol & Transcript ID & Log2 units (strong VS weak) & $\mathrm{p}$-value & Category \\
\hline$\overline{F 13 A 1}$ & NM_204685 & -0.949 & 0.0234 & EM \\
\hline FREM1 & XM_424932 & -0.910 & 0.0057 & EM \\
\hline MEGF10 & XM_424719 & 3.910 & 0.0398 & EM \\
\hline MGAT4C & XM_425447 & 3.007 & 0.0497 & $\mathrm{GM}$ or $\mathrm{CM}$ \\
\hline CHST3 & NM_205121 & 2.839 & 0.0372 & $\mathrm{GM}$ or $\mathrm{CM}$ \\
\hline EDEM3 & XM_422293 & 2.539 & 0.0179 & $\mathrm{GM}$ or $\mathrm{CM}$ \\
\hline$L A R G E$ & NM_001004404 & 2.071 & 0.0429 & $\mathrm{GM}$ or $\mathrm{CM}$ \\
\hline GFPT2 & XM_424573 & 1.919 & 0.0173 & $\mathrm{GM}$ or $\mathrm{CM}$ \\
\hline GALNTL1 & XM_001231964 & 1.895 & 0.0452 & GM or $\mathrm{CM}$ \\
\hline WDR77 & NM_001030916 & 1.805 & 0.0445 & $\mathrm{GM}$ or $\mathrm{CM}$ \\
\hline NDST3 & XM_426325 & 1.403 & 0.0121 & $\mathrm{GM}$ or $\mathrm{CM}$ \\
\hline$B 3 G A L T 1$ & XM_426584 & 1.254 & 0.0483 & $\mathrm{GM}$ or $\mathrm{CM}$ \\
\hline$O G D H L$ & XM_421503 & 1.144 & 0.0022 & GM or $\mathrm{CM}$ \\
\hline MAN1A2 & XM_416490 & -4.700 & 0.0087 & $\mathrm{GM}$ or $\mathrm{CM}$ \\
\hline POFUT2 & XM_421892 & -2.804 & 0.0156 & GM or $\mathrm{CM}$ \\
\hline LOC772154 & XM_001235329 & -2.798 & 0.0006 & GM or $\mathrm{CM}$ \\
\hline$K L B$ & XM_423224 & -2.335 & 0.0380 & $\mathrm{GM}$ or $\mathrm{CM}$ \\
\hline TRIM7.2 & NM_001099354 & -1.986 & 0.0041 & GM or $\mathrm{CM}$ \\
\hline LOC771361 & XM_001234647 & -1.433 & 0.0369 & $\mathrm{GM}$ or $\mathrm{CM}$ \\
\hline RCJMB04_28l23 & NM_001039316 & -1.324 & 0.0034 & $\mathrm{GM}$ or $\mathrm{CM}$ \\
\hline NDST4 & XM_420638 & -1.239 & 0.0261 & $\mathrm{GM}$ or $\mathrm{CM}$ \\
\hline$P F K M$ & NM_204223 & -1.170 & 0.0460 & $\mathrm{GM}$ or $\mathrm{CM}$ \\
\hline NUP153 & XM_418937 & -1.109 & 0.0378 & GM or $\mathrm{CM}$ \\
\hline$G P D 1 L$ & XM_418763 & -0.947 & 0.0383 & $\mathrm{GM}$ or $\mathrm{CM}$ \\
\hline PHKA2 & XM_416811 & -0.926 & 0.0326 & $\mathrm{GM}$ or $\mathrm{CM}$ \\
\hline B3GNTL1 & XM_415599 & -0.883 & 0.0109 & $\mathrm{GM}$ or $\mathrm{CM}$ \\
\hline$P M M 1$ & XM_416228 & -0.787 & 0.0490 & $\mathrm{GM}$ or $\mathrm{CM}$ \\
\hline$M M P 11$ & XM_001232776 & 2.209 & 0.0391 & $\mathrm{GM}$ or $\mathrm{CM}$ \\
\hline ST3GALA & XM_417860 & -1.043 & 0.0094 & GM or $\mathrm{CM}$ \\
\hline
\end{tabular}

IT represents ion/proton transporter, EM represents extracellular matrix, GM represents post-translation glycosylation modification, and CM represents carbohydrate metabolism

As shown in Table 4 and Table 5, many DE-transcripts are involved in signal transduction, such as GO terms purinergic nucleotide receptor activity (GO:0001614), nucleotide receptor activity (GO:0016502), purinergic receptor activity (GO:0035586), transmembrane signaling receptor activity (GO:0004888), and negative regulation of BMP signaling pathway (GO:0030514). Among them, GO:0004888 dominantly contains 33 transcripts encoding signal receptors, and these receptors could be further classified into several subgroups: OXTR, LOC431251 and SSTR3 belong to reproductive hormone receptors; $C H R M 2$, ADRA2B, P2RX4, P2RY2, EDNRB2, GABRB2, GABRG2, LOC428961 and NPFFR2 function as receptors mediating neurotransmitters or neuropeptide; GRIN2B and GRIN3A could modulate the efficiency of synaptic transmission; NTRK1 and NTRK2 belong to the receptor tyrosine kinase (RTK) family, and are involved with neurotrophin (GO:0005030 - neurotrophin receptor activity; and GO:0043121 - neurotrophin binding) (Table 5).
Besides various enriched molecular function shown above, many biophysical processes are also found to be enriched among the DE-transcripts, including a series of processes and subgroups (Tables 4 and 5). GO:0003951 ( $\mathrm{NAD}^{+}$kinase activity) modulate the metabolism or redox in cell (Table 5). Enrichment of GO:0009409 (response to cold) may reflect the fact the rearing condition of experimental hens was in the winter at room temperature about 2 to $10^{\circ} \mathrm{C}$. GO:0046209 (nitric oxide metabolic process) may regulate vascular or smooth muscle relaxation or other functions. GO:0002028 is involved in ion transportation. While the subgroup processes of muscular development and activity include skeletal muscle fiber development (enrichments of GO:0048741, GO:0048747 and GO:0055002) and striated muscle contraction regulation (enrichments of GO:0055117 and GO:0006942). It is of note that there is almost no striated muscle in avian uterus except smooth muscle. However, the chicken genome project was completed in 2004, and the functional 
Table 4. Enriched gene ontology (GO) terms revealed from identified DE-transcripts according to biological_process ontology

\begin{tabular}{|c|c|c|c|c|}
\hline Group & GOID & Term & $\mathrm{p}$ & Gene symbol or representative public ID \\
\hline \multirow[t]{3}{*}{$\begin{array}{l}\text { Reproductive hormone } \\
\text { synthesis and regulation }\end{array}$} & GO:0060126 & $\begin{array}{l}\text { Somatotropin secreting cell } \\
\text { differentiation }\end{array}$ & 0.013 & OTX2, WNT4 \\
\hline & GO:0021984 & $\begin{array}{l}\text { Adenohypophysis } \\
\text { development }\end{array}$ & 0.044 & OTX2, WNT4 \\
\hline & GO:0032355 & Response to estradiol stimulus & 0.044 & SOCS2, AREGB \\
\hline Signal transduction & GO:0030514 & $\begin{array}{l}\text { Negative regulation of BMP } \\
\text { signaling pathway }\end{array}$ & 0.030 & TOB1, GREM1 \\
\hline \multirow[t]{12}{*}{ Biophysical processes } & GO:0048741 & $\begin{array}{l}\text { Skeletal muscle fiber } \\
\text { development }\end{array}$ & 0.012 & $S L C 23 A 2, C H A T$ \\
\hline & GO:0015074 & DNA integration & 0.012 & LOC770294, LOC770705, ENS-3 \\
\hline & GO:0055117 & $\begin{array}{l}\text { Regulation of cardiac muscle } \\
\text { contraction }\end{array}$ & 0.013 & $P 2 R X 4, N K X 2-5$ \\
\hline & GO:0009409 & Response to cold & 0.018 & $I L 4, S L C 27 A 1$ \\
\hline & GO:0048747 & Muscle fiber development & 0.022 & SLC23A2, CHAT \\
\hline & GO:0046209 & $\begin{array}{l}\text { Nitric oxide metabolic } \\
\text { process }\end{array}$ & 0.024 & $P 2 R X 4, C P S 1$ \\
\hline & GO:0007586 & Digestion & 0.030 & PGA5, PRSS2, LOC396365 \\
\hline & GO:0015849 & Organic acid transport & 0.033 & $\begin{array}{l}\text { SLC23A2, OCA2, LOC770309, } \\
\text { SLC7A14, SLC27A1 }\end{array}$ \\
\hline & GO:0046942 & Carboxylic acid transport & 0.033 & $\begin{array}{l}\text { SLC23A2, OCA2, LOC770309, } \\
\text { SLC7A14, SLC27A1 }\end{array}$ \\
\hline & GO:0055002 & $\begin{array}{l}\text { Striated muscle cell } \\
\text { development }\end{array}$ & 0.034 & SLC23A2, CHAT, TTN, NKX2-5 \\
\hline & GO:0006942 & $\begin{array}{l}\text { Regulation of striated muscle } \\
\text { contraction }\end{array}$ & 0.037 & $P 2 R X 4, N K X 2-5$ \\
\hline & GO:0002028 & $\begin{array}{l}\text { Regulation of sodium ion } \\
\text { transport }\end{array}$ & 0.044 & $N K X 2-5, N E D D 4 L$ \\
\hline \multirow[t]{8}{*}{$\begin{array}{l}\text { Reproductive biophysical } \\
\text { processes }\end{array}$} & GO:0060748 & $\begin{array}{l}\text { Tertiary branching involved } \\
\text { in mammary gland duct } \\
\text { morphogenesis }\end{array}$ & 0.009 & $W N T 4, A R$ \\
\hline & GO:0060745 & $\begin{array}{l}\text { Mammary gland branching } \\
\text { involved in pregnancy }\end{array}$ & 0.013 & $W N T 4, A R$ \\
\hline & GO:0060562 & $\begin{array}{l}\text { Epithelial tube } \\
\text { morphogenesis }\end{array}$ & 0.019 & $\begin{array}{l}\text { DEAF1, WNT3, GREM1, WNT4, } \\
\text { NKX2-5, HOXA11, AR, AREGB }\end{array}$ \\
\hline & GO:0060444 & $\begin{array}{l}\text { Branching involved in } \\
\text { mammary gland duct } \\
\text { morphogenesis }\end{array}$ & 0.020 & $W N T 4, A R, A R E G B$ \\
\hline & GO:0009994 & Oocyte differentiation & 0.024 & WNT4, GDF9 \\
\hline & GO:0048599 & Oocyte development & 0.024 & WNT4, GDF9 \\
\hline & GO:0060603 & $\begin{array}{l}\text { Mammary gland duct } \\
\text { morphogenesis }\end{array}$ & 0.033 & $W N T 4, A R, A R E G B$ \\
\hline & GO:0060135 & $\begin{array}{l}\text { Maternal process involved in } \\
\text { female pregnancy }\end{array}$ & 0.037 & $W N T 4, A R$ \\
\hline
\end{tabular}

GOID represents the identifiers, and Term represents term definitions for Gene Ontology term entities. p: p-value of significance (Welch t-test).

gene database of $G$. gallus remains incomplete, some ontology annotations of DE-genes may refer to mammalian homologs, which may account for our results. The genes related to muscular cell contraction are likely to modulate the mobility of uterus to facilitate egg rotation and calcification (Johnson, 1986; Jonchère et al., 2010). Similarly, there is no digestion process in the uterus, three genes in GO:0007586 (digestion process), PGA5 (an aspartic acid protease, which is involved in ovulation (Peluffo et al., 2011)), PRSS2 and LOC396365 (preprogastrin), are likely to promote the maturation of secretary extracellular proteins or regulate the secretion of uterus glands and mobility of uterus.

The final group of reproductive biophysical processes also includes several subgroups of processes (Table 4). Epithelial tube morphogenesis (GO:0060562) may regulate 
Table 5. Enriched gene ontology (GO) terms revealed from identified DE-transcripts according to molecular function ontology

\begin{tabular}{|c|c|c|c|c|}
\hline Group & GOID & Term & $\mathrm{p}$ & Gene symbol or representative public ID \\
\hline \multirow[t]{6}{*}{ Signal transduction } & GO:0005030 & $\begin{array}{l}\text { Neurotrophin receptor } \\
\text { activity }\end{array}$ & 0.013 & NTRK1, NTRK2 \\
\hline & GO:0001614 & $\begin{array}{l}\text { Purinergic nucleotide } \\
\text { receptor activity }\end{array}$ & 0.017 & $P 2 R X 4, P 2 R Y 2$, ENSGALG00000012080 \\
\hline & GO:0016502 & $\begin{array}{l}\text { Nucleotide receptor } \\
\text { activity }\end{array}$ & 0.017 & $P 2 R X 4, P 2 R Y 2$, ENSGALG00000012080 \\
\hline & GO:0043121 & Neurotrophin binding & 0.024 & NTRK1, NTRK2 \\
\hline & GO:0035586 & $\begin{array}{l}\text { Purinergic receptor } \\
\text { activity }\end{array}$ & 0.026 & $P 2 R X 4, P 2 R Y 2$, ENSGALG00000012080 \\
\hline & GO:0004888 & $\begin{array}{l}\text { Transmembrane signaling } \\
\text { receptor activity }\end{array}$ & 0.049 & $\begin{array}{l}\text { OXTR, LOC431251, SSTR } 3, \text { CHRM2, } \\
\text { ADRA2B, P2RX4, P2RY2, EDNRB2, } \\
\text { GABRB2, GABRG2, LOC428961, } \\
\text { NPFFR2, GRIN2B, GRIN3A, NTRK1, } \\
\text { NTRK2, EPHB6, DDR2, TMPRSS6, } \\
\text { PCSK5, CCKAR, IFNAR2, CSF1R, } \\
\text { TLR5, OR10A7, LOC768958, } \\
\text { LOC769317, LOC777484, GPR39, } \\
\text { GPR97, ENSGALG00000017405, } \\
\text { ENSGALG00000017093, } \\
\text { ENSGALG00000012080. }\end{array}$ \\
\hline \multirow[t]{2}{*}{ Biophysical processes } & GO:0003951 & NAD+ kinase activity & 0.013 & C5orf $33, N A D K$ \\
\hline & GO:0005319 & Lipid transporter activity & 0.049 & $\begin{array}{l}\text { ATP11C, ATP8A2, ATP8B3, APOB, } \\
L O C 769564, \text { SLC27A1 }\end{array}$ \\
\hline
\end{tabular}

GOID represents the identifiers, and Term represents term definitions for gene ontology term entities. p: p-value of significance (Welch t-test).

the development of uterus glands (tubular epithelial glands). Oocyte development subgroup contains oocyte differentiation (GO:0009994) and oocyte development (GO:0048599). Female pregnancy subgroup contains enrichments of GO:0060135, GO:0060745, GO:0060748, GO:0060444 and GO:0060603.

Overall, laying is an avian reproductive behavior, and eggshell calcification is regulated by relative reproductive hormones and neurotransmitters, which may finally affect eggshell quality through a complex suite of biophysical reactions.

\section{Confirmation of DE-transcripts by qRT-PCR}

21 DE-transcripts (9 up-regulated and 12 downregulated) were chosen for validation using qRT-RCR experiments, and the four microarray samples were tested in pairs for \#19 vs \#35 and \#40 vs \#80, respectively. As shown in Figure 3, 16 out of the 21 tested transcripts (76\%) were confirmed by qRT-PCR experiments, though the absolute fold-change values are slightly different. The remaining transcripts, CRYBB1, EXOC6B, LOC416916, MAN1A2 and CHST3, showed inconsistent differential expression between qRT-PCR and microarray experiments.

GAPDH, CHST3, GALNTL1, NDST4, LARGE, SP1, RHOBTB2, and WDR72 were selected to examine the PCR efficiency. The results showed the PCR efficiency of these genes ranged from $86.8 \%$ to $94.2 \%$, and the PCR efficiency of inner reference $(G A P D H)$ and other genes seemed nearly similar.

\section{DISCUSSION}

Laying is regarded as avian reproductive behavior, which is regulated by reproductive hormones and neurotransmitters. The chicken oviduct has been extensively used as a model to study hormonal induction of protein synthesis (Khuong and Jeong. 2011). Under the control of steroid hormones or neurotransmitters, the tubular gland epithelial cells synthesize and secrete a great variety of proteins to form egg white and eggshell when egg passes through the oviduct (Mann et al., 2006). In this paper, 1,195 DE-transcripts have been identified to be related with eggshell strength. GOEAST analysis further identify some significantly enriched GO terms, and the enriched GO terms suggest that some DE-transcripts mediate reproductive hormones or neurotransmitters to affect eggshell quality (Tables 4 and 5).

Both terms GO:0060126 and GO:0021984 are involved in reproductive hormone regulation, and share two genes, OTX2 and WNT4.

Otx2 is a paired-like homeodomain transcription factor, which can mediate GnRH (gonadotropin releasing hormone) signaling (Kelley et al., 2000). Functional studies revealed that Otx2 is required as early as gastrulation for neural induction, and even for brain development (Rhinn et al., 1998). However, Otx2 is also of importance for 


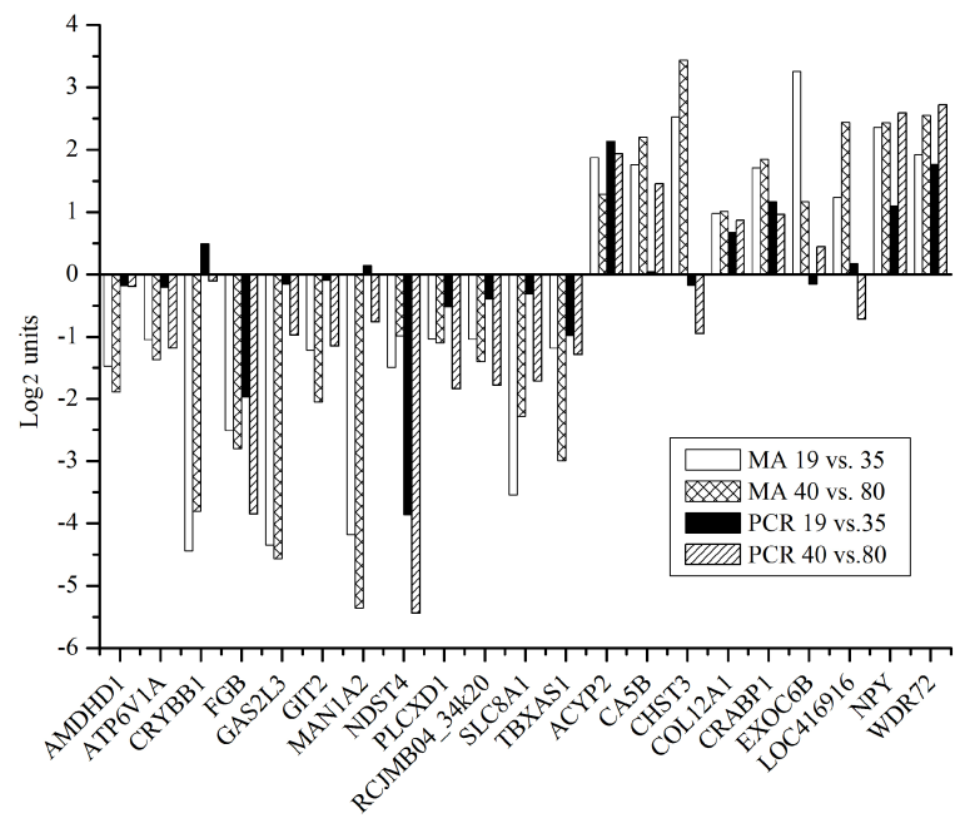

Figure 3. Real-time RT-PCR validation of microarray data. Expression levels of the first 12 transcripts (AMDHD1, ATP6V1A, CRYBB1, FGB, GAS2L3, GIT2, MAN1A2, NDST4, PLCXD1, RCJMB04-34k20, SLC8A1 and TBXAS1) were down-regulated in microarray experiment, and the last 9 transcripts (ACYP2, CA5B, CHST3, COL12A1, CRABP1, EXOC6B, LOC416916, NPY and WDR72) were up-regulated in microarray experiment. Among the above transcripts, five transcripts (CRYBB1, MAN1A2, CHST3, EXOC6B and LOC416916) failed to be verified by real-time RT-PCR.

neurogenesis and cellular proliferation in multiple other tissues (Layman et al., 2011).

As a member of the WNT family, Wnt4 is a secreted glycoprotein signaling molecule and involved in paracrine signaling (Diaz et al., 2011). Wnt4 is critical for female sex determination and differentiation (Chen et al., 2011). In the female, Wnt4 is positively involved in ovarian development; while in the male mutated WNT4 will result in aberrant testis development (Diaz et al., 2011; Barrionuevo et al., 2012). On the other hand, Wnt4 is also potent to regulate the development of the female reproductive tract (Franco et al., 2011). Furthermore, WNT4 is expressed postnatally in ovarian follicles and corpora lutea, and its expression increases in response to gonadotropin (Hsieh et al., 2002). Wnt4 mediates follicle development and fertility by regulating the expression of genes involved in steroidogenesis, prostaglandin biosynthesis, tissue remodeling, and angiogenesis (Hsieh et al., 2002; Boyer et al., 2010). Moreover, excluding its reproductive contributions, WNT4 is also tightly associated with bone strength (Zmuda et al., 2011).

Our results also show that some DE-transcripts are involved in signal transduction (Tables 4 and 5), among which, NTRK1, NTRK2, P2RX4, and P2RY2 are overlapped in multiple enriched GO terms (Table 5).

Ntrk1, also named TrkA, and Ntrk2 TrkB, are two members of the neurotrophic tyrosine kinase receptor (NTKR) family. These kinases are membrane-bound receptors mediating various functions of neurotrophins, such as cell survival, migration, outgrowth of axons and dendrites, synaptogenesis, remodeling of synapses, and synaptic transmission (Ohira1 and Hayashi, 2009). So far, several neurotrophins have been well studied, such as nerve growth factor (NGF), brain-derived neurotrophic factor (BDNF), neurotrophin 3 (NT-3), and NT-4/5. NTKRs are high affinity receptors of neurotrophins. TrkA mediates the biological response of NGF, while BDNF and NT-4/5 are the preferred ligands for TrkB (Ohiral and Hayashi, 2009). Additionally, NTKRs also play roles in some biomaterials. NT-4 may modulate proliferation and differentiation of the dental epithelium and promote production of the enamel matrix via the TrkB-MAPK pathway (Yoshizaki et al., 2008).

Both $\mathrm{P} 2 \mathrm{RX} 4$ and $\mathrm{P} 2 \mathrm{RY} 2$ are purinergic receptors. Purinergic receptors are subdivided into metabotropic P2Y receptors and ionotropic $\mathrm{P} 2 \mathrm{X}$ receptors. $\mathrm{P} 2 \mathrm{Y}$ receptors are coupled to G-protein and trigger inositol 1,4,5-triphosphate (IP3)-induced intracellular $\mathrm{Ca}^{2+}$ release following activation of phospholipase $\mathrm{C}$, while $\mathrm{P} 2 \mathrm{X}$ receptors are ligand-gated ion channels. P2RX4 will be discussed later, while P2RY2 performs a dominant role in calcium signaling during osteoblast differentiation (Nishii et al., 2009). It is known that extracellular ATP, UTP, and PPi can strongly block the mineralization of matrix nodules, while this potent inhibition of bone formation is mediated by P2RY2 (Orriss et al., 2007). Furthermore, P2RY2 is also involved in 
inhibition of intercellular communication between osteoblasts (Hoebertz et al., 2003).

At present, there are at least three cDNA microarray studies globally investigating the gene expression in chicken shell gland (Yang et al., 2007; Dunn et al., 2009; Jonchère et al., 2010), but the overlap among the DE-genes from these studies is not plentiful. Different animals, tissue samples or treatment methods may partially account for this problem.

Yang et al. (2007) harvested uterus tissues at $2 \mathrm{~h}$ post oviposition, and screened out 34 known genes in the shell glands between hens with low and high egg productive rates. This study and our data share a single gene CALD1 (caldesmon 1) (Figure 4). CALD1 is a ubiquitous actin and calmodulin binding protein, and functions as a substrate for mitogen-activated protein kinase (Childs et al., 1992) or as serine and threonine kinases (Sutherland et al., 1994).

Dunn et al. (2009) identified 266 DE-genes in shell glands from 25-week old mature hens comparing to 12 week old juveniles from high and low bone quality lines, respectively. The tissues sampled when eggs passed through the oviducts but not in shell glands. Three DEgenes are also found in our data: NADK (NAD kinase), LOC422993 (Similar to interferon-induced membrane protein Leu-13/9-27), and LAMP3 (lysosomal-associated membrane protein 3) (Figure 4), suggesting potential crucial function of these genes in not only early stage of eggshell calcification but also other stages of eggshell formation.

Jonchère et al. (2010) used the 40-week old hens at $18 \mathrm{~h}$ post oviposition (corresponding to the rapid phase of calcification), and identified 469 DE-known genes in uterus versus both white isthmus, and magnum. There are 7 genes consistently identified in their study and our data, such as $P 2 R X 4$ (purinergic receptor $\mathrm{P} 2 \mathrm{X}$, ligand-gated ion channel, 4), FSTL1 (follistatin-like 1), TUBGCP4 (Tubulin, gamma complex associated protein 4), WDR77 (WD repeat domain 77), RCJMB04_6g16 (microtubule-associated protein 1 light chain 3 beta), PWP1 (PWP1 homolog in S. cerevisiae) and $S G K 1$ (serum/glucocorticoid regulated kinase 1) (Figure 4).

On the other hand, three additional DE-genes in our data were previously found in the acid soluble part of chicken eggshell organic matrix (Mann et al., 2006). These three genes, FSTL1 (follistatin-like 1), CAMK2D (calcium/ calmodulin-dependent protein kinase (CaM kinase) II delta) and KRT75 (keratin 75) (Figure 4), could reflect potential interaction of eggshell calcification and organic matrix formation.

Among these overlapping DE-genes, both $P 2 R X 4$ (Jonchère et al., 2010) and NADK (Yang et al., 2007) are also present in our enriched GO terms (Tables 4 and 5), and FSTL1 (Mann et al., 2006; Jonchère et al., 2010) occurs in more than three relative studies.

$\mathrm{P} 2 \mathrm{RX} 4$ is one member of the $\mathrm{P} 2 \mathrm{X}$ receptors ( $\mathrm{P} 2 \mathrm{RX})$. $\mathrm{P} 2 \mathrm{RX}$ are ionotropic ATP-gated ion channels conducting $\mathrm{Ca}^{2+}$ inflow (Fodor et al., 2009), with high capability of $\mathrm{Ca}^{2+}$ permeabilities corresponding to at least 100 -fold those of $\mathrm{Na}^{+}$(Burnashev, 1998). In chondrogenic mesenchymal

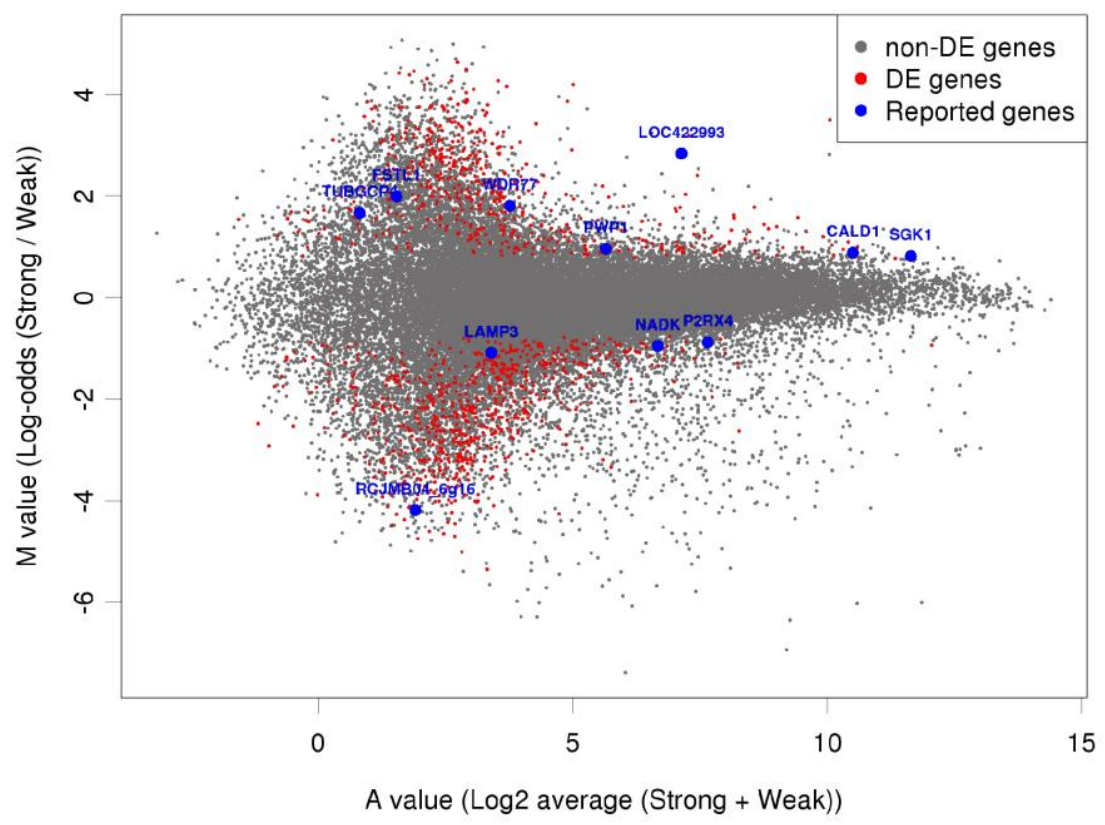

Figure 4. MA-plot of all microarray tested chicken-genes. X-axis: the average normalized expression values across all 4 (strong+weak) eggshell samples (in $\log _{2}$ scale); Y-axis: the log-odds ratio between the average expression values of strong vs. weak eggshell samples; grey dots: nondifferentially expressed genes (non-DE genes); red dots: differentially expressed genes (DE-genes) identified in this study; big blue dots: DE-genes (CALD1, NADK, LOC422993, LAMP3, P2RX4, FSTL1, TUBGCP4, WDR77, RCJMB04_6g16, PWP1 and $S G K 1)$ reported in previous studies. 
cells, $\mathrm{P} 2 \mathrm{X} 4$ receptors could conduct $\mathrm{Ca}^{2+}$ inflow to elevate intracellular $\mathrm{Ca}^{2+}$ levels, and finally promoting extracellular matrix production (Fodor et al., 2009). Eggshell calcification requires considerable ion transportation, especially $\mathrm{Ca}^{2+}$, and various extracellular matrix synthesis and secretion, whether and how P2RX4 channels regulate these processes requires further studies.

NAD kinases (NADKs) are a family of enzymes transferring a phosphate group from ATP to NAD to generate and maintain the cellular NADP pool (Pollak et al., 2007). It is reported, that during development of placenta, the expression level of $N A D K$ appears drastically elevated (Lerner et al., 2001).

Fstl1 is a secreted glycoprotein belonging to the BM40/SPARC/osteonectin family containing both calciumbinding domain and Follistatin-like domain (Hambrock et al., 2004). As a mesenchymal factor, Fstl1 is critical for oviduct development, and determines the differentiation of secretary epithelial cells and ciliated epithelial cells in the oviduct (Umezu et al., 2010). This means Fstl1 may modulate chicken endometrium development during eggshell formation. However, Fstl1 is also present in the organic part of eggshells (Mann et al., 2006), and Jonchère et al. (2010) propose it may be a uterine antiprotease

\section{IMPLICATIONS}

Above all, using Affymetrix Chicken Array, 1,195 DEtranscripts were identified in the shell gland between "high shell strength" and "low shell strength" hens, which represent 941 unique known genes. According to gene ontology annotations, these transcripts are involved in a wide range of biological processes; the most prominent DEtranscripts relate to signal transduction, metabolism, extracellular matrix, or ion transport and homeostasis, and so on. Furthermore, Gene Ontology (GO) term enrichment of DE-transcripts suggests that avian eggshell calcification is likely to be regulated by relative reproductive hormones and neurotransmitters, which may finally affect eggshell quality through a complex suite of biophysical processes.

\section{ACKNOWLEDGEMENTS}

This work was supported by National Natural Science Foundation of China (grant no. 30700567) and Zhejiang Provincial Natural Science Foundation of China (grant no. LY12C17002).

\section{REFERENCES}

Ahmed, A. M. H., A. B. Rodriguez-Navarro, M. L. Vidal, J. Gautron, J. M. Garcia-Ruiz and Y. Nys. 2005. Changes in eggshell mechanical properties, crystallographic texture and in matrix proteins induced by moult in hens. Br. Poult. Sci. 46:268-279.

Barrionuevo, F. J., M. Burgos, G. Scherer and R. Jiménez. 2012. Genes promoting and disturbing testis development. Histol. Histopathol. 27:1361-1383.

Burnashev, N. 1998. Calcium permeability of ligand-gated channels. Cell Calcium. 24:325-332.

Boyer, A., É. Lapointe, X. Zheng, R. G. Cowan, H. Li, S. M. Quirk, F. J. DeMayo, J. S. Richards, and D. Boerboom. 2010. WNT4 is required for normal ovarian follicle development and female fertility. FASEB J. 24:3010-3025.

Carrino, D. A., J. P. Rodriguez and A. I. Caplan. 1997. Dermatan sulfate proteoglycans from the mineralized matrix of the avian eggshell. Connect. Tissue Res. 36:175-193.

Chen, B., P. Suo, B. Wang, J. Wang, L. Yang, S. Zhou, Y. Zhu, X. Ma and Y. Cao. 2011. Mutation analysis of the WNT4 gene in Han Chinese women with premature ovarian failure. Reprod. Biol. Endocrinol. 9:75.

Cheuk, B. L. and S. W. Cheng. 2011. Differential expression of elastin assembly genes in patients with Stanford Type A aortic dissection using microarray analysis. J. Vasc. Surg. 53:10711078.

Childs, T. J., M. H. Watson, J. S. Sanghera, D. L. Campbell, S. L. Pelech and A. S. Mak. 1992. Phosphorylation of smooth muscle caldesmon by mitogen-activated protein (MAP) kinase and expression of MAP kinase in differentiated smooth muscle cells. J. Biol. Chem. 267:22853-22859.

Creger, C. R., H. Phillips and J. T. Scott. 1976. Formation of an eggshell. Poult. Sci. 55:1717-1723.

Diaz, F. J., K. Anthony and A. N. Halfhill. 2011. Early avian follicular development is characterized by changes in transcripts involved in steroidogenesis, paracrine signaling and transcription. Mol. Reprod. Dev. 78:212-223.

Dunn, I. C., P. W. Wilson, Z. Lu, M. M. Bain, C. L. Crossan, R. T. Talbot and D. Waddington. 2009. New hypotheses on the function of the avian shell gland derived from microarray analysis comparing tissue from juvenile and sexually mature hens. Gen. Comp. Endocrinol. 163:225-232.

Fodor, J., C. Matta, T. Juhász, T. Oláh, M. Gönczi, Z. Szíjgyártó, P. Gergely, L. Csernoch and R. Zákány. 2009. Ionotropic purinergic receptor $\mathrm{P} 2 \mathrm{X} 4$ is involved in the regulation of chondrogenesis in chicken micromass cell cultures. Cell Calcium. 45:421-430.

Franco, H. L., D. Dai, K. Y. Lee, C. A. Rubel, D. Roop, D. Boerboom, J. W. Jeong, J. P. Lydon, I. C. Bagchi, M. K. Bagchi and F. J. Demayo. 2011. WNT4 is a key regulator of normal postnatal uterine development and progesterone signaling during embryo implantation and decidualization in the mouse. FASEB J. 25:1176-1187.

Gautron, J., M. T. Hincke, K. MANN, M. Panhéleux, M. Bain, M. D. McKee, S. E. Solomon and Y. Nys. 2001a. Ovocalyxin-32, a novel chicken eggshell matrix protein: Isolation, amino acid sequencing, cloning and immunocytochemical localization. J. Biol. Chem. 276:39243-39252.

Gautron, J., M. T. Hincke, M. Panhéleux, J. M. Garcia-Ruiz, T. Boldicke and Y. Nys. 2001b. Ovotransferrin is a matrix protein of the hen eggshell membranes and basal calcified layer. Connect. Tissue Res. 42:255-267.

Gautron, J., E. Murayama, A. Vignal, M. Morisson, M. D. McKee, 
S. Réhault, V. Labas, M. Belghazi, M. L Vidal, Y. Nys and M. T. Hincke. 2007. Cloning of Ovocalyxin-36, a novel chicken eggshell protein related to lipopolysaccharide-binding proteins (LBP), bactericidal permeability-increasing proteins (BPI), and plunc family proteins. J. Biol. Chem. 282:5273-5286.

Greenfield, E. M., D. C. Wilson and M. A. Crenshaw. 1984 Ionotropic nucleation of calcium carbonate by molluscan matrix. Amer. Zool. 24:925-932.

Hambrock, H. O., B. Kaufmann, S. Müller, F. G. Hanisch, K. Nose, M. Paulsson, P. Maurer and U. Hartmann. 2004. Structural characterization of TSC-36/Flik: Analysis of two charge isoforms. J. Biol. Chem. 279:11727-11735.

Hincke, M. T. 1995. Ovalbumin is a component of the chicken eggshell matrix. Connect. Tissue Res. 31:227-233.

Hincke, M. T., J. Gautron, M. Panhéleux, J. Garcia-Ruiz, M. D. McKee and Y. Nys. 2000. Identification and localization of lysozyme as a component of eggshell membranes and eggshell matrix. Matrix Biol. 19:443-453.

Hincke, M. T., C. P. Tsang, M. Courtney, V. Hill and R. Narbaitz. 1995. Purification and immunochemistry of a soluble matrix protein of the chicken eggshell (Ovocleidin 17). Calcif. Tissue Int. 56:578-583.

Hsieh, M., M. A. Johnson, N. M. Greenberg and J. S. Richards. 2002. Regulated expression of Wnts and Frizzleds at specific stages of follicular development in the rodent ovary. Endocrinology. 143:898-908.

Johnson, A. L. 1986. Reproduction in the female. In: Avian Physiology. 4th ed. (Ed. P. D. Sturkie). Springer-Verlag, New York..

Jonchère, V., S. Réhault-Godbert, C. Hennequet-Antier, C. Cabau, V. Sibut, L. A Cogburn, Y. Nys and J. Gautron. 2010. Gene expression profiling to identify eggshell proteins involved in physical defense of the chicken egg. BMC Genomics. 11:5775.

Kelley, C. G., G. Lavorgna, M. E. Clark, E. Boncinelli and P. L. Mellon. 2000. The Otx2 homeoprotein regulates expression from the gonadotropin-releasing hormone proximal promoter. Mol. Endocrinol. 14:1246-1256.

Khuong, T. T. and D. K. Jeong. 2011. Adipogenic differentiation of chicken epithelial oviduct cells using only chicken serum. In Vitro Cell. Dev. Biol. Anim. 47:609-614.

Lakshminarayanan, R., R. M. Kini and S. Valiyaveettil. 2002. Investigation of the role of ansocalcin in the biomineralization in goose eggshell matrix. Proc. Natl. Acad. Sci. U.S.A. 99:5155-5159.

Lavelin, I., N. Meiri, M. Einat, O. Genina and M. Pines. 2002. Mechanical strain regulation of the chicken glypican-4 gene expression in the avian eggshell gland. Am. J. Physiol. Regul. Integr. Comp. Physiol. 283:R853-R861.

Lavelin, I., N. Yarden, S. Ben-Bassat, A. Bar and M. Pines. 1998. Regulation of osteopontin gene expression during egg shell formation in the laying hen by mechanical strain. Matrix Biol. 17:615-623

Layman, W. S., E. A. Hurd and D. M. Martin. 2011. Reproductive dysfunction and decreased GnRH neurogenesis in a mouse model of CHARGE syndrome. Hum. Mol. Genet. 20:31383150 .

Lerner, F., M. Niere, A. Ludwig and M. Ziegler. 2001. Structural and functional characterization of human NAD kinase.
Biochem. Biophys. Res. Commun. 288:69-74

Mann, K. 1999. Isolation of a glycosylated form of the chicken eggshell protein ovocleidin and determination of the glycosylation site. Alternative glycosylation/phosphorylation at an N-glycosylation sequon. FEBS Lett. 463:12-14.

Mann, K., J. Gautron, Y. Nys, M. D. McKee, T. Bajari, W. J. Schneider and M. T. Hincke. 2003. Disulfide-linked heterodimeric clusterin is a component of the chicken eggshell matrix and egg white. Matrix Biol. 22:397-407.

Mann, K., B. Maček and J. V. Olsen. 2006. Proteomic analysis of the acid-soluble organic matrix of the chicken calcified eggshell layer. Proteomics. 6:3801-3810.

Mann, K., J. V. Olsen, B. Maček, F. Gnad and M. Mann. 2007. Phosphoproteins of the chicken eggshell calcified layer. Proteomics. 7:106-115.

Nishii, N., N. Nejime, C. Yamauchi, N. Yanai, K. Shinozuka and T. Nakabayashi. 2009. Effects of ATP on the intracellular calcium level in the osteoblastic TBR31-2 cell line. Biol. Pharm. Bull. 32:18-23.

Nys, Y., J. Gautron, J. M. Garcia-Ruiz and M. T. Hincke. 2004. Avian eggshell mineralization: biochemical and functional characterization of matrix proteins. C. R. Palevol. 3:549-562.

Ohira, K. and M. Hayashi. 2009. A new aspect of the TrkB signaling pathway in neural plasticity. Curr. Neuropharmacol. 7:276-285.

Orriss, I. R., J. C. Utting, A. Brandao-Burch, K. Colston, B. R. Grubb, G. Burnstock and T. R. Arnett. 2007. Extracellular nucleotides block bone mineralization in vitro: Evidence for dual inhibitory mechanisms involving both P2Y2 receptors and pyrophosphate. Endocrinology 148:4208-4216.

Peluffo, M. C., M. J. Murphy, S. T. Baughman, R. L. Stouffer and J. D. Hennebold. 2011. Systematic analysis of protease gene expression in the rhesus macaque ovulatory follicle: metalloproteinase involvement in follicle rupture. Endocrinology 152:3963-3974.

Pines, M., V. Knopov and A. Bar. 1995. Involvement of osteopontin in egg shell formation in the laying chicken. Matrix Biol. 14:765-771.

Pollak, N., M. Niere and M. Ziegler. 2007. NAD kinase levels control the NADPH concentration in human cells. J. Biol. Chem. 282:33562-33571.

Reyes-Grajeda, J. P., A. Moreno and A. Romero. 2004. Crystal structure of ovocleidin-17, a major protein of the calcified Gallus gallus eggshell. J. Biol. Chem. 279:40876-40881.

Ramakers, C., J. M. Ruijter, R. H. Deprez, A. F. Moorman. 2003. Assumption-free analysis of quantitative real-time polymerase chain reaction (PCR) data. Neurosci. Lett. 339:62-66.

Rhinn, M., A. Dierich, W. Shawlot, R. R. Behringer, M. Le Meur and S. L. Ang. 1998. Sequential roles for Otx2 in visceral endoderm and neuroectoderm for forebrain and midbrain induction and specification. Development. 125:845-856.

Ruijter, J. M., C. Ramakers, W. M. Hoogaars, Y. Karlen, O. Bakker, M. J. van den Hoff, A. F. Moorman. 2009. Amplification efficiency: linking baseline and bias in the analysis of quantitative PCR data. Nucleic Acids Res. 37:e45e45.

Sauveur, B. and M. Reviers. 1988. Reproduction des volailles et production d'œufs. INRA Editions, Paris.

Solomon, S. E. 1999. Gordon Memorial Lecture. An egg ist ein ei, 
es un huevo, est un oeuf. Br. Poult. Sci. 40:5-11.

Sutherland, C., B. S. Renaux, D. J. Mckay and M. P. Walsh. 1994. Phosphorylation of caldesmon by smooth-muscle casein kinase II. J. Muscle Res. Cell Motil. 15:440-456.

Umezu, T., H. Yamanouchi, Y. Iida, M. Miura and Y. Tomooka. 2010. Follistatin-like-1, a diffusible mesenchymal factor determines the fate of epithelium. Proc. Natl. Acad. Sci. USA. 107:4601-4606.

Veis, A. 1989. Chemical and biochemical perspectives. in: Biomineralization (Ed. S. Mann, J. Webb and R. J. P. Williams) pp. 189. VCH, Weinhein, New York.

Yang, K. T., C. Y. Lin, J. S. Liou, Y. H. Fan, S. H. Chiou, C. W. Huang, C. P. Wu, E. C. Lin, C. F. Chen, Y. P. Lee, W. C. Lee, S. T. Ding, W. T. Cheng and M. C. Huang. 2007. Differentially expressed transcripts in shell glands from low and high egg production strains of chickens using cDNA microarrays. Anim. Reprod. Sci. 101:113-124.
Yoshizaki, K., S. Yamamoto, A. Yamada, K. Yuasa, T. Iwamoto, E. Fukumoto, H. Harada, M. Saito, A. Nakasima, K. Nonaka, Y. Yamada and S. Fukumoto. 2008. Neurotrophic factor neurotrophin-4 regulates ameloblastin expression via fulllength TrkB. J. Biol. Chem. 283:3385-3391.

Zheng, Q. and X. J. Wang. 2008. GOEAST: a web-based software toolkit for Gene Ontology enrichment analysis. Nucleic Acids Res. 36:358-363.

Zmuda, J. M., L. M. Yerges-Armstrong, S. P. Moffett, L. Klei, C. M. Kammerer, K. Roeder, J. A. Cauley, A. Kuipers, K. E. Ensrud, C. S. Nestlerode, A. R. Hoffman, C. E. Lewis, T. F. Lang, E. Barrett-Connor, R. E. Ferrell, E. S. Orwoll and Osteoporotic Fractures in Men (MrOS) Study Group. 2011. Genetic analysis of vertebral trabecular bone density and cross-sectional area in older men. Osteoporos Int. 22:10791090 . 